\title{
TITLE: A non-toxic dose of cobalt chloride blocks hair cells of the zebrafish lateral line
}

\author{
William J. Stewart ${ }^{1}$, Jacob L. Johansen ${ }^{1} \&$ James C. Liao ${ }^{1 *}$
}

${ }^{1}$ Department of Biology, Whitney Laboratory for Marine Bioscience, University of Florida, St. Augustine, FL, USA.

*Correspondence to: jliao@whitney.ufl.edu

Key words: Lateral line; cobalt chloride; zebrafish; hair cells.

Abstract: Experiments on the flow-sensitive lateral line system of fishes have provided important insights into the function and sensory transduction of vertebrate hair cells. A common experimental approach has been to pharmacologically block lateral line hair cells and measure how behavior changes. Cobalt chloride $\left(\mathrm{CoCl}_{2}\right)$ blocks the lateral line by inhibiting calcium movement through the membrane channels of hair cells, but high concentrations can be toxic, making it unclear whether changes in behavior are due to a blocked lateral line or poor health. Here, we identify a non-toxic treatment of cobalt that completely blocks lateral line hair cells. We exposed 5-day post fertilization zebrafish larvae to $\mathrm{CoCl}_{2}$ concentrations ranging from 1-20 $\mathrm{mM}$ for 15 minutes and measured 1) the spiking rate of the afferent neurons contacting hair cells and 2) the larvae's health and long-term survival. Our results show that a 15-minute exposure to $5 \mathrm{mM} \mathrm{CoCl}_{2}$ abolishes both spontaneous and evoked afferent firing. This treatment does not change swimming behavior, and results in $>85 \%$ survival after 5 days. Weaker treatments of $\mathrm{CoCl}_{2}$ did not eliminate afferent activity, while stronger treatments caused close to $50 \%$ mortality. Our work provides a guideline for future zebrafish investigations where physiological confirmation of a blocked lateral line system is required.

Short title: Cobalt blocks zebrafish hair cells 


\section{Introduction.}

The flow-sensitive lateral line system of fishes is composed of a series of mechanoreceptor clusters distributed across the surface of the body. Each cluster, called a neuromast, consists of hair cells that detect information about the surrounding fluid (e.g., pressure and velocity). Experiments which block the hair cells of the lateral line have revealed the crucial role of the lateral line in capturing prey (Coombs and Conley, 1997), evading predators (Stewart et al., 2014), and navigating and orienting to current (Windsor et al., 1998; Montgomery et al., 1997). Blocking occurs when the afferent neurons contacting hair cells stop firing, which is typically achieved by exposing fish to aminoglycoside antibiotics (Harris et al., 2003; Ton and Parng, 2005; Owens et al., 2009; Van Trump et al., 2010), such as neomycin and gentamycin, or divalent cations such as cobalt (Karlsen and Sand, 1987). Aminoglycoside antibiotics are ototoxic and block the lateral line by triggering hair cell death (Huth et al., 2011). Recovery of function involves the regeneration of the affected hair cells (McHenry et al., 2009).

Divalent cations like cobalt can block the lateral line by competitively inhibiting calcium from entering the hair cells through voltage-gated channels of the cell membrane (Karlsen and Sand, 1987), which halts calcium-dependent synaptic vesicle fusion and neurotransmitter release to stop communication with downstream afferent neurons. In high concentrations, cobalt can also trigger cell death after entering the hair cells of the lateral line (Becker, 2013) or the mammalian cochlea (Li et al., 2015; Lee et al., 2016). The mechanisms for the death of hair cells and associated neurons from high concentrations of cobalt exposure are poorly understood. In rats, we know that cobalt has the potential to become toxic by acting as an inhibitor of zinc-dependent 
enzymes (Hartwig, 2001). Other deleterious effects may be caused by its activity as a calcium channel antagonist, which promotes the inhibition of calcium entry and calcium signaling during critical cellular activities (Barnes and Hille, 1989; Akbar et al., 2011). Prolonged or high-dose treatment with cobalt chloride switches cell metabolism from aerobic respiration to anaerobic glycolysis (Regazzetti et al., 2009). This induction of oxidative stress can provoke cell death through the increase in expression of the highly toxic superoxide anion in hair cells and of caspase-3 (Jomova and Valko, 2011; Lee et al., 2013; Huk et al., 2004).

Treatments of both antibiotics and divalent cations can be toxic to fish at high doses (Kaus, 1987; Janssen, 2000). Therefore, the main goal of this paper is to identify a dose of cobalt that blocks hair cells of the lateral line without compromising the overall health of the animal. This is important because interpretations of behavioral changes after treatment should be attributed to the loss of lateral line function, and not a systemic response to a toxic over dose. Here, we provide a recommendation for safely blocking lateral line hair cells with cobalt chloride $\left(\mathrm{CoCl}_{2}\right)$ by measuring how cobalt affects 1$)$ the spiking activity of afferent neurons in response to direct stimulation of connected neuromasts and 2) the long-term behavior and survival of fish. Zebrafish larvae were chosen for this study because they provide a particularly strong system in which to investigate the neural circuitry of a tractable hair cell system in vivo, and are a widely-used model system in the fields of development, genetics, and neuroscience.

\section{Material and methods.}

2.1 Measuring the effect of cobalt on survival. We evaluated how $\mathrm{CoCl}_{2}$ concentration affected the survival of zebrafish larvae. 15 groups of 20 healthy, 5 days post fertilization larvae $(N=$ 300) were randomly assigned one of five treatments (control, 1, 5, 10, and $20 \mathrm{mM} \mathrm{CoCl}_{2}$ in 
Hank's solution). The larvae were exposed to their respective treatments for 15 minutes and the number of surviving larvae was recorded at 5, 10, and 15 minutes during treatment. The larvae were then transferred to dishes containing Hank's solution and their survival was monitored for 5 days.

2.2 Measuring how cobalt affects the sensitivity of the lateral line. We next evaluated how the concentrations of $\mathrm{CoCl}_{2}$ used in our survival experiments affected the firing of the afferent neurons connected to neuromasts. We did this by measuring both the spontaneous and evoked spiking rates of afferent neurons with electrophysiology. Each fish was paralyzed $(0.1 \% \alpha-$ bungarotoxin) and secured to a dish containing $3 \mathrm{~mL}$ of extracellular solution (XCL, $134 \mathrm{mM}$ $\mathrm{NaCl}, 2.9 \mathrm{mM} \mathrm{KCl}, 1.2 \mathrm{mM} \mathrm{MgCl} 2,2.1 \mathrm{mM} \mathrm{CaCl}_{2}, 10 \mathrm{mM}$ glucose, $10 \mathrm{mM}$ HEPES buffer, adjusted to a $\mathrm{pH}$ of 7.8 with $\mathrm{NaOH}$ ). Extracellular, loose patch recordings were made from single afferent neurons in the posterior lateral line ganglion, as previously (Haehnel-Taguchi et al., 2014). During the recording, we deflected the connected neuromast with a $30 \mathrm{~Hz}$ sine wave stimulus for 10 seconds using a glass probe driven by a piezoelectric actuator. The rounded end of the probe $(\sim 300 \mu \mathrm{m}$ diameter $)$ was positioned $\sim 100 \mu \mathrm{m}$ to the side of the neuromast and was driven back and forth over a distance of $150 \mu \mathrm{m}$ (Fig. 1a). We then removed $1.5 \mathrm{~mL}$ of XCL from the dish and carefully perfused a $1.5 \mathrm{~mL}$ solution of concentrated $\mathrm{CoCl}_{2}$ in $\mathrm{XCL}$ (or XCL alone for control experiments) as appropriate to bring the final concentration to the desired treatment level. After mixing in the $\mathrm{CoCl}_{2}$ solution, the afferent response to the stimulus was immediately measured, and then again at two minute intervals for 20 minutes. Preceding each stimulation, we also recorded the spontaneous spiking for at least two seconds. Experiments were conducted on 5 larvae for each treatment. All data were analyzed in Matlab. 


\subsection{Measuring the behavior of zebrafish larvae after cobalt treatment. Our survival experiments}

and electrophysiological recordings allowed us to identify the optimal treatment of $\mathrm{CoCl}_{2}$. We next sought to determine how long this optimal treatment blocked the lateral line. In our hands, it was not possible to maintain patch recordings of afferent neurons long enough to document the recovery of spikes after cobalt washout. Therefore, we determined the functional recovery of the lateral line by measuring the probability that treated larvae responded to a small fluid jet during the days following exposure. 100 larvae (5 days post fertilization) were exposed to the optimal treatment of $\mathrm{CoCl}_{2}$ and equally divided into 25 petri dishes. Each larva was carefully exposed to fluid jets generated manually with a pipette (McHenry et al., 2010). This stimulus was chosen for its efficacy and to minimize vestibular cues caused by whole-body displacement of the animal. The opening of the pipette was positioned $\sim 10 \mathrm{~mm}$ beside a larva and 3 small and sequential jets were aimed at the animal. Dye visualizations before experiments showed that each jet produced a small vortex ring that collided with the animal. Larvae that responded to the jet executed an obvious and rapid escape maneuver. All larvae were exposed to these jets for 14 times over the first 5 hours after treatment, and then once a day for 5 days. To further verify that the lateral line mediates the responses to the fluid jet, we measured the ability of 20 larvae ( 5 dpf) to respond to fluid jets before and after a 1-hour treatment with $250 \mu \mathrm{m}$ neomycin sulfate. This dosage has been demonstrated to reliably ablate lateral line hair cells (McHenry et al., 2009). We found that the probability of response decreased sharply from 0.95 to 0.05 following the neomycin treatment, as expected.

To evaluate how the optimal treatment affected the health of the zebrafish larvae, we measured spontaneous swimming before and after treatment. 50 larvae were placed together in a large petri dish. After 30 minutes to allow acclimation, a 1 minute video of the larvae was 
digitally recorded to measure the probability of spontaneous swimming. All larvae were then treated with $\mathrm{CoCl}_{2}$ and the probability of spontaneous swimming was measured 7 times over the next 5 hours. As a control, we repeated these measurements on an additional set of 50 untreated fish.

\section{Results}

All animals survived the initial 15-minute exposure to all $\mathrm{CoCl}_{2}$ treatment concentrations (0-20 $\mathrm{mM} \mathrm{CoCl} 2$ ), but there was mortality in each group after 5 days (Fig. $2 a$ ). Survivability was optimal at the 1 and $5 \mathrm{mM}$ concentrations, with at least $85 \%$ of the larvae surviving. However, for concentrations above $10 \mathrm{mM}$, only about $50 \%$ of larvae survived. All individuals from the control group survived.

Cobalt quickly decreased the sensitivity of the lateral line afferent neurons to neuromast stimulation (Fig. 1b). The spiking rate of afferent neurons decreased within minutes for all treatments (Fig. 2b), with higher concentrations of $\mathrm{CoCl}_{2}$ causing the spiking rate to decrease more rapidly (decay coefficient, Fig. $2 c$ ). Fifteen minutes of exposure to $1 \mathrm{mM} \mathrm{CoCl} 2$ decreased the evoked spike rate over $40 \%$, while 5 and $10 \mathrm{mM} \mathrm{CoCl}_{2}$ effectively abolished it. The evoked firing rate from the control group did not change over 20 minutes. We found that the synchronization of the spikes to deflection (i.e., vector strength, Fig. $2 d$ ) was the lowest for 10 $\mathrm{mM} \mathrm{CoCl}_{2}$, and exhibited the highest variation for $1 \mathrm{mM} \mathrm{CoCl}$.

Exposure to high concentrations of $\mathrm{CoCl}_{2}$ quickly abolished spontaneous spiking in afferent neurons. After only 4 minutes of exposure to 5 and $10 \mathrm{mM} \mathrm{CoCl}_{2}$, spontaneous activity was, on average, absent from afferent neurons (Fig. $2 e$ ). For $1 \mathrm{mM} \mathrm{CoCl}_{2}$, we observed wide 
fluctuations of the spontaneous spike rate, at times increasing considerably above, but never below, control levels. The spontaneous activity of afferent neurons from the control group did not change over time.

Larvae recovered their ability to sense flow one day after $\mathrm{CoCl}_{2}$ treatment. We measured the probability that larvae treated with $5 \mathrm{mM} \mathrm{CoCl}_{2}$ responded to a fluid jet, and found that the probability of a response slowly increased after treatment (regression, $\mathrm{P}<0.001$ ) and fully recovered to control levels after 24 hours $(N=100$, Fig $2 a)$. Larvae also swam normally after this treatment, suggesting that the concentration was non-toxic $(N=50$, Fig. $3 b)$.

\section{Discussion}

Blocking the lateral line system with antibiotics or $\mathrm{CoCl}_{2}$ has yielded important insights into the function of vertebrate hair cell systems, yet high concentrations of either can be toxic to larval and adult fish (Janssen, 2000). Based on our results, we recommend exposing zebrafish larvae to $5 \mathrm{mM} \mathrm{CoCl}_{2}$ for 15 minutes to block the lateral line; this treatment completely disables the lateral line but still ensures over $85 \%$ survival after 5 days. Since treated larvae slowly recover their ability to sense flow, we suggest that experiments are conducted within an hour after treatment. While the time course of lateral-line recovery for both $\mathrm{CoCl}_{2}$ and antibiotics is similar (McHenry et al., 2010), recovery of function occurs through cellular mechanisms in intact hair cells in the former. In the latter, function is recovered through the generation of new hair cells. We can imagine experiments where dose-dependent, graded blocking of the lateral line hair cells is preferred over promoting hair cell death with antibiotics. Such studies might include investigating intrinsic afferent and efferent contact dynamics with hair cells, or the contribution of afferent spike rate on motor behaviors. This work shows that treating fish with cobalt is an 
effective and safe method for blocking lateral line hair cells, similar to antibiotic treatments (Harris et al., 2003). However, our electrophysiological recordings of afferent neurons during exposure to $\mathrm{CoCl}_{2}$ provide detailed, dose-dependent insight into how an ototoxic drug blocks the lateral line in vivo. Exposure to cobalt provides an alternative technique to future investigators requiring high confidence in a safe ablation protocol that has been physiologically confirmed.

Regardless of how the lateral line is pharmacologically blocked, the ideal technique should completely block the lateral line while still ensuring that the animal remains healthy. It is well documented that cobalt is toxic at high concentrations (Janssen, 2000), and this toxicity depends on both the duration of exposure and the species of fish. For example, a continuous 12hour exposure to low concentrations $(0.01-0.03 \mathrm{mM})$ is non-lethal and can block the lateral line in adult Rutilus rutilus (Karlsen and Sand, 1987), but higher concentrations (1 mM) have been shown to lead to substantial mortality. In Astyanax mexicanus, a 3-hour exposure to $2 \mathrm{mM} \mathrm{CoCl}_{2}$ caused complete mortality as soon as 17 hours post-treatment (Janssen, 2000). This is important because behavioral changes due to exposure to high concentrations of $\mathrm{CoCl}_{2}$ may be interpreted as a consequence of a blocked lateral line, when in fact it may be due to the compromised health of the animal. For cavefish, high concentrations of $\mathrm{CoCl}_{2}$ indeed elicit changes in swimming behavior that are health-related and not specific to the lateral line (Montgomery et al., 1997; Janssen, 2000). Our work indicates that larval zebrafish can tolerate higher concentrations of $\mathrm{CoCl}_{2}$ than the adults of other species since $5 \mathrm{mM} \mathrm{CoCl}_{2}$ did not affect spontaneous swimming (Fig. $3 b$ ) and caused only $15 \%$ mortality after 5 days.

The function of the lateral line is fully recovered after exposure to cobalt or antibiotics. In the present study, larvae regained their sensitivity to a fluid jet 24 hours after cobalt treatment (Fig. 3a). Similarly, zebrafish larvae treated with $250 \mu \mathrm{M}$ neomycin for $1 \mathrm{hr}$ fully recovered their 
ability to respond to rapid suction after 24 hours, even though hair cells required 55 hours to regenerate (McHenry et al., 2009). Larvae treated with $200 \mu \mathrm{M}$ neomycin for 1 hour required a longer recovery of 52 hours before they could properly orient themselves to a current flow (Suli et al., 2012), and Ma et al. (2008) shows recovery of lateral line morphology at $48-72 \mathrm{hrs}$ postneomycin. This variation illustrates that the time course of recovery depends on the criteria in which recovery is evaluated in addition to the method of treatment.

The cessation of spontaneous activity in afferent neurons does not indicate that the lateral line is functionally blocked. During exposure to 5 and $10 \mathrm{mM} \mathrm{CoCl}_{2}$, spontaneous activity of afferent neurons stopped after 4 minutes. However, we found the afferent neurons still responded to direct stimulation for up to 15 minutes (Fig 1b). This stresses the importance of using electrophysiology to verify when the lateral line is completely blocked by $\mathrm{CoCl}_{2}$. Spontaneous afferent activity in an untreated fish results from calcium entering the resting hair cells through "leaky" channels (i.e, voltage-gated calcium channels that have a non-zero open probability at resting membrane potentials [Trapani and Nicolson, 2011]), which triggers the release of glutamate and afferent spiking. We believe that 1 and $5 \mathrm{mM} \mathrm{CoCl}_{2}$ can quickly and completely block these open voltage-gated calcium channels. However, upon hair cell deflection many more channels open, likely resulting in a longer time period required for $\mathrm{CoCl}_{2}$ to abolish evoked responses.

Note that exposure to $1 \mathrm{mM} \mathrm{CoCl}_{2}$ caused distinct oscillations in spontaneous spike rate. We hypothesize that additional calcium channels open in hair cells as others are blocked by cobalt, producing these temporary increases in spontaneous activity. Such opening of residual calcium channels is inundated by high concentrations of $\mathrm{CoCl}_{2}$, where an excess of cobalt ions 
quickly blocks any new open voltage-gated calcium channels. High $\mathrm{CoCl}_{2}$ concentrations thus eliminate spontaneous activity regardless of any homeostatic responses.

Our in vivo electrophysiological recordings of afferent neuron activity characterize how different concentrations of $\mathrm{CoCl}_{2}$ disable hair cells in healthy zebrafish. Our results are poised to contribute to future work investigating the transduction physiology of hair cells in this widelyused model species.

We have no competing interests.

J. C. Liao conceived and supervised the study. W. Stewart carried out the electrophysiology recordings, the behavioral experiments, and analyzed the data. J. Johansen carried out the survival experiments and analyzed the resultant data. J. C. Liao and W. Stewart wrote the manuscript with comments from J. Johansen.

All protocols were approved by the University of Florida Institutional Animal Care and Use Committee.

This research was supported by the National Institutes of Health RO1 DC010809 and NSF IOS 1257150 to J.C.L. We would like to thank Bill and Martha Dobes for their generosity in the aftermath of Hurricane Matthew.

\section{References}


Akbar,M.,Brewer,J.,Grant,M.,2011.Effect of chromium and cobalt ions on primary human lymphocytes in vitro. Journal of Immunotoxicology 8, 140-149.

Barnes,S.,Hille,B.,1989.Ionic channels of the inner segment of tiger salamander cone photoreceptors. Journal of General Physiology 94, 719-743.

Becker,E.A.,2013.The distribution and ontogeny of neuromast receptor organs and a comparison of methods for chemical ablation of the lateral line system in two cichlid fishes. Masters thesis, The University of Rhode Island.

Coombs,S.,Conley,R.A.,1997.Dipole source localization by the mottled sculpin II. The role of lateral line excitation patterns. Journal of Comparative Physiology A 180, 401-415.

Hassan,E.S.,Abdel-Latif,H.,Biebricher,R.,1992.Studies on the effects of Ca2++ and Co++ on the swimming behavior of the blind Mexican cave fish. Journal of Comparative Physiology A 171, 413-419.

Janssen,J.,2000.Toxicity of Co2+: implications for lateral line studies. Journal of Comparative Physiology A 186, 957-960.

Jomova,K.,Valko,M.,2011.Advances in metal-induced oxidative stress and human disease. Toxicology 283, 65-87.

Haehnel-Taguchi,M., Akanyeti,O., Liao,J.C.,2014.Afferent and motoneuron activity in response to single neuromast stimulation in the posterior lateral line of larval zebrafish. Journal of neurophysiology 112, 1329-1339.

Harris,J.A.,Cheng,A.G.,Cunningham,L.L.,MacDonald,G.,Raible,D.W.,Rubel,E.W.,2003. Neomycin-induced hair cell death and rapid regeneration in the lateral line of zebrafish (Danio rerio). Journal of the Association for Research in Otolaryngology, 4, 219-234.

Hartwig,A.,2001.Zinc finger proteins as potential targets for toxic metal ions: differential effects on structure and function. Antioxidants and Redox Signaling, 3, 625-634.

Huk,O.,Catelas,I.,Mwale,F.,Antoniou,J.,Zukor,D.,Petit,A.,2004.Induction of apoptosis and necrosis by metal ions in vitro. Journal of Arthroplasty 19,84-87.

Huth,M.E.,Ricci,A.J.,Cheng,A.G.,2011.Mechanisms of aminoglycoside ototoxicity and targets of hair cell protection. International journal of otolaryngology 2011, 1-20.

Karlsen,H.E.,Sand,O.,1987.Selective and reversible blocking of the lateral line in freshwater fish. Journal of Experimental Biology 133, 249-262.

Kaus,S.1987.The effect of aminoglycoside antibiotics on the lateral line organ of Aplocheilus lineatus (Cyprinodontidae). Acta oto-laryngologica 103, 291-298. 
Lee,J. et al.,2013. $\mathrm{CoCl}_{2}$ induces apoptosis through the mitochondria- and death receptormediated pathway in the mouse embryonic stem cells. Molecular and Cellular Biochemistry 379, $133-140$.

Lee,J.N.,et al.,2016.3-Aminotriazole protects from $\mathrm{CoCl}_{2}$-induced ototoxicity by inhibiting the generation of reactive oxygen species and proinflammatory cytokines in mice. Archives of toxicology, 90, 78-791.

Li,P.,Ding,D.,Salvi,R.,Roth,J.A.,2015.Cobalt-induced ototoxicity in rat postnatal cochlear organotypic cultures. Neurotoxicity research, 28, 209-221.

Ma,E.,Rubel,E.,Raible,D.,2008.Notch signaling regulates the extent of hair cell regeneration in the zebrafish lateral line. Journal of Neuroscience, 28, 2261-2273.

McHenry,M.J.,Feitl,K.E.,Strother,J.A.,Van Trump,W.J., 2009. Larval zebrafish rapidly sense the water flow of a predator's strike. Biology Letters 5, 477-479.

McHenry,M.J.,Michel,K.B.,Stewart,W.,Müller,U.K.,2010.Hydrodynamic sensing does not facilitate active drag reduction in the golden shiner (Notemigonus crysoleucas). Journal of Experimental Biology, 213, 1309-1319.

Montgomery,J.C.,Baker,C.F.,Carton,A.G.,1997.The lateral line can mediate rheotaxis in fish. Nature 389, 96-963.

Owens,K.N.,Coffin,A.B.,Hong,L.S.,Bennett,K.O.C.,Rubel,E.W.,Raible,D.W.,2009.Response of mechanosensory hair cells of the zebrafish lateral line to aminoglycosides reveals distinct cell death pathways. Hearing research, 253, 32-41.

Regazzetti,C,.Peraldi,P.,Gremeaux,T. et al.,2009.Hypoxia decreases insulin signaling pathways in adipocytes. Diabetes 58, 95-103.

Stewart,W.J.,Cardenas,G.S.,McHenry,M.J.,2013.Zebrafish larvae evade predators by sensing water flow. Journal of Experimental Biology 216, 388-398.

Suli,A.,Watson,G.M.,Rubel,E.W.,Raible,D.W., 2012. Rheotaxis in larval zebrafish is mediated by lateral line mechanosensory hair cells. PloS one, 7, 29727.

Ton,C.,Parng,C.,2005.The use of zebrafish for assessing ototoxic and otoprotective agents. Hearing research, 208, 79-88.

Trapani,J.G.,Nicolson,T.,2011.Mechanism of spontaneous activity in afferent neurons of the zebrafish lateral-line organ. The Journal of Neuroscience 31, 1614-1623.

Van Trump,W.J.,Coombs,S.,Duncan,K.,McHenry,M.J.,2010.Gentamicin is ototoxic to all hair cells in the fish lateral line system. Hearing research 261, 42-50. 
Windsor,S.P.,Tan,D.,Montgomery,J.C.,2008.Swimming kinematics and hydrodynamic imaging in the blind Mexican cave fish (Astyanax fasciatus). Journal of Experimental Biology 211, 29502959.

\section{Figure Legends}

Figure 1. Afferent activity before and during exposure to $15 \mathrm{mM} \mathrm{CoCl}_{2}$. (a), A glass probe was used to stimulate a neuromast at $30 \mathrm{~Hz}$ while the activity of the connected afferent neuron was recorded with extracellular loose-patch recordings. (b), Blue traces show the spontaneous and evoked afferent activity before treatment and the minutes during exposure to $15 \mathrm{mM} \mathrm{CoCl}$.

Figure 2. The effects of $\mathrm{CoCl}_{2}$ on the zebrafish lateral line system and survival. $(a)$, The percent of larvae surviving a 15-minute exposure to different concentrations of $\mathrm{CoCl}_{2}$. $(b)$, The evoked afferent spike rate in response to direct neuromast deflection (30 Hz sine wave stimulus) under different $\mathrm{CoCl}_{2}$ treatments. Time 0 indicates the beginning of exposure. Data from 1, 5, and 10 $\mathrm{mM} \mathrm{CoCl} l_{2}$ treatments were fit with an 2-parameter exponential curve function $\left(y=a e^{b x}\right)$, where shaded regions indicate the $95 \%$ prediction intervals for each fit. For the control treatment, the horizontal line and shaded region shows the mean spike rate $\pm 1 \mathrm{SD}$, respectively. $(c)$, The coefficients of decay for the exponential curve fits from $(b)$. Error bars indicate $95 \%$ confidence intervals. $(d)$, The mean vector strength of evoked spikes. Error bars show \pm 1 SD. $(e)$, The spontaneous spike rate under different $\mathrm{CoCl}_{2}$ treatments. Error bars show $\pm 1 \mathrm{SD}$. In $(b)$ and $(e)$, the values are normalized to the spike rate measured at time 0 for each treatment. 
Figure 3. The behavior of zebrafish larvae after exposure to $5 \mathrm{mM} \mathrm{CoCl}_{2}$ for 15 minutes. (a), The probability that control and treated larvae responded to a fluid jet after treatment. $(b)$, The probability that treated and control larvae spontaneously swim during one-minute periods after treatment. Shaded regions show the $95 \%$ confidence intervals of the probability estimate. 
A

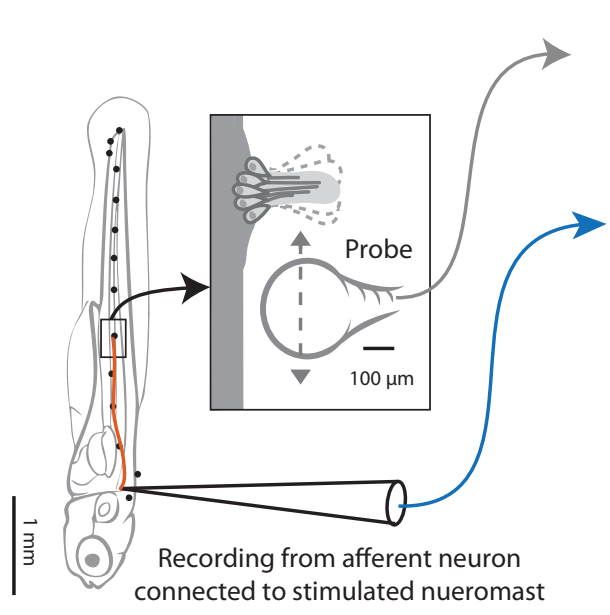

B

$30 \mathrm{~Hz}$

stimulation

spontaneous activity

before treatment

4 min of
exposure
8 min of
exposure

exposure

12 min of exposure

$16 \mathrm{~min}$ of exposure

e

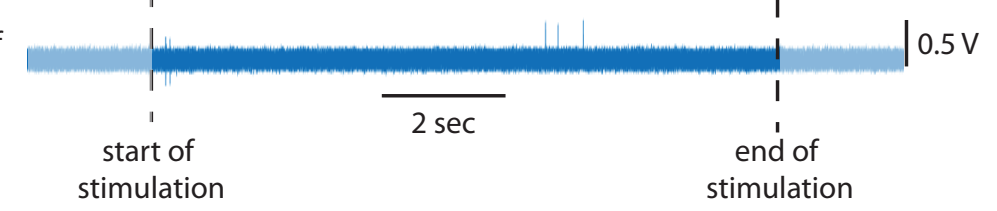


(a)

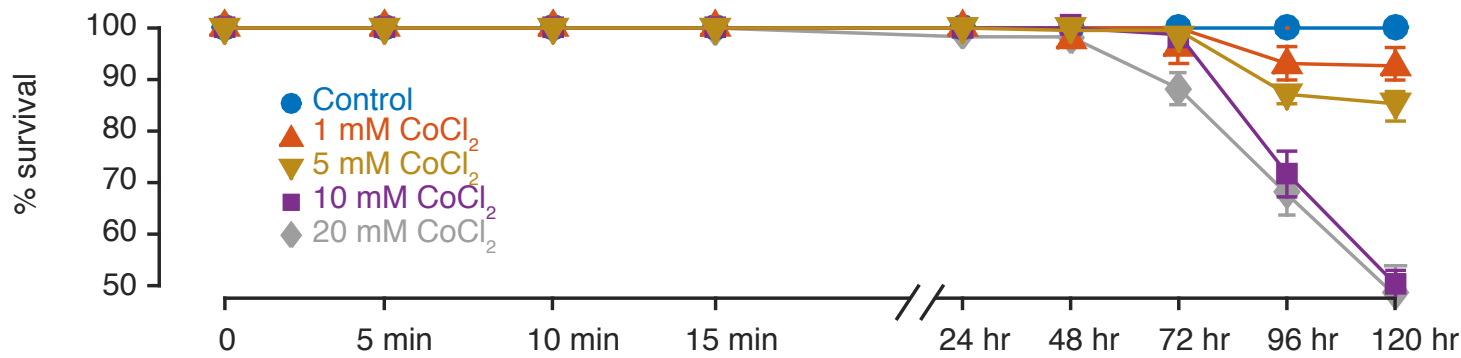

(b)
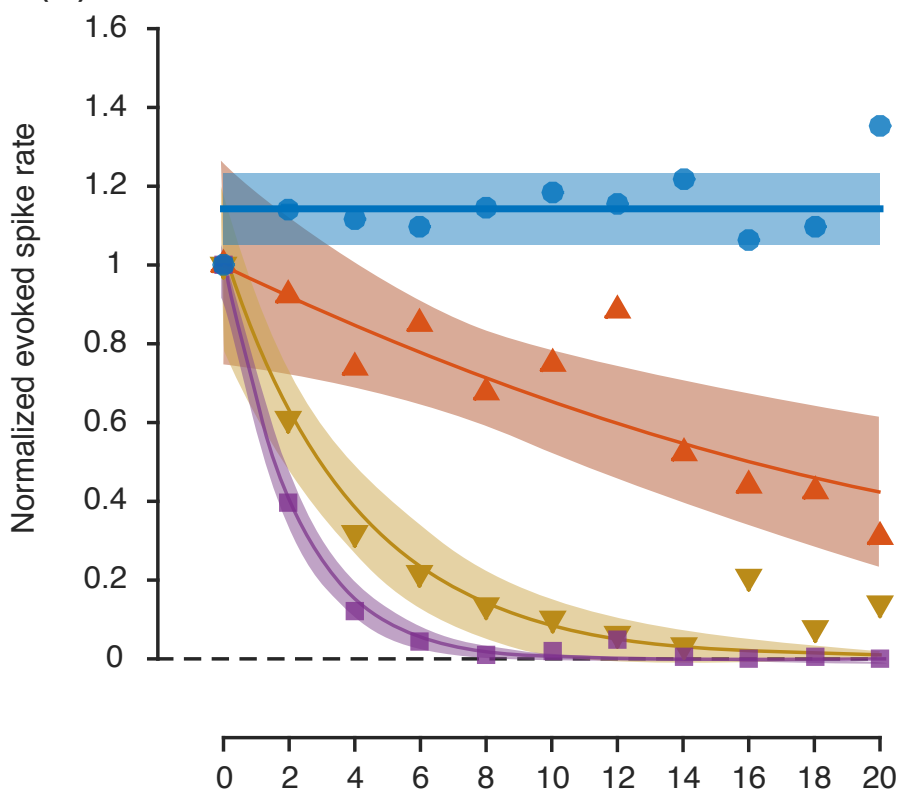

(e)

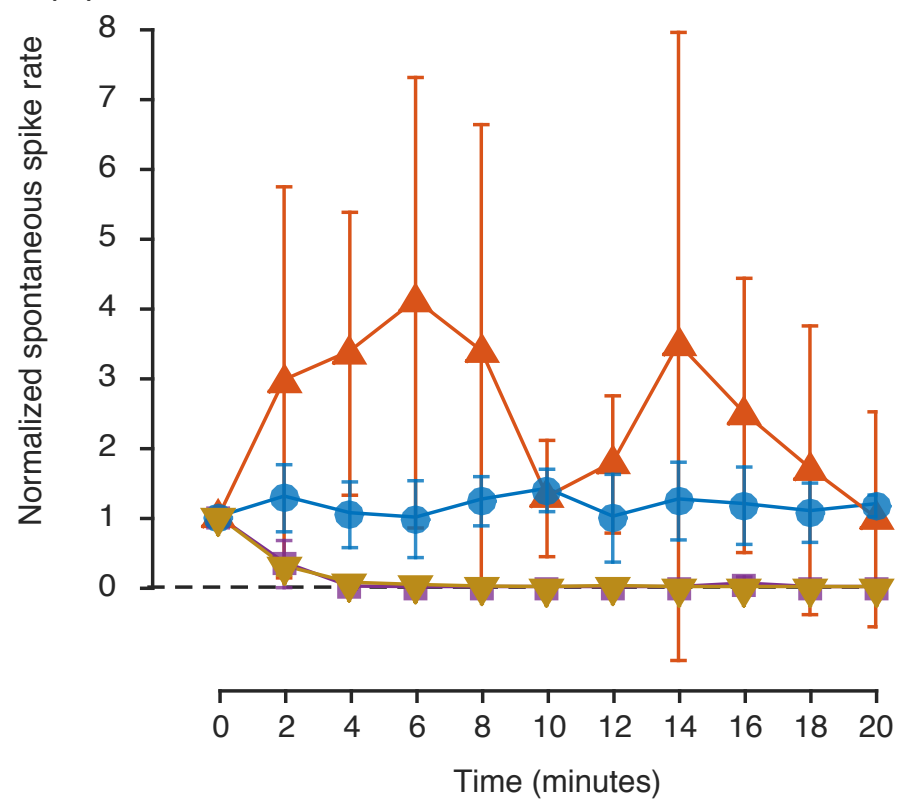

(c)

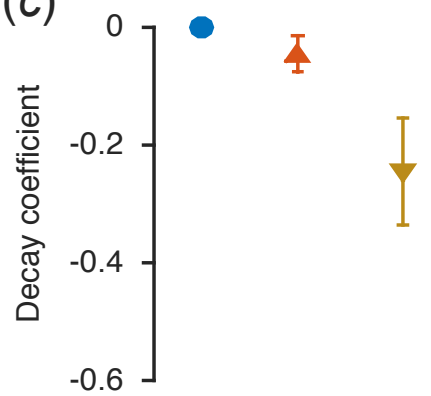

1

(d)

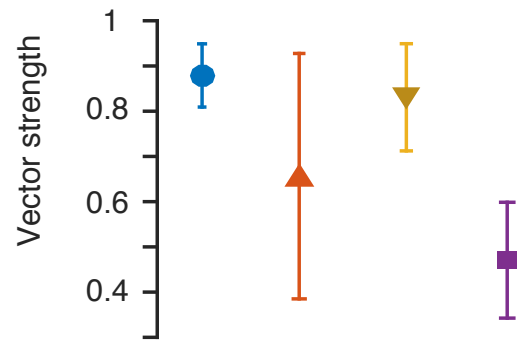

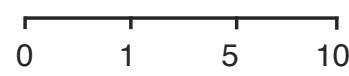

$\mathrm{CoCl}_{2}$ concentration (mM) 
(a)
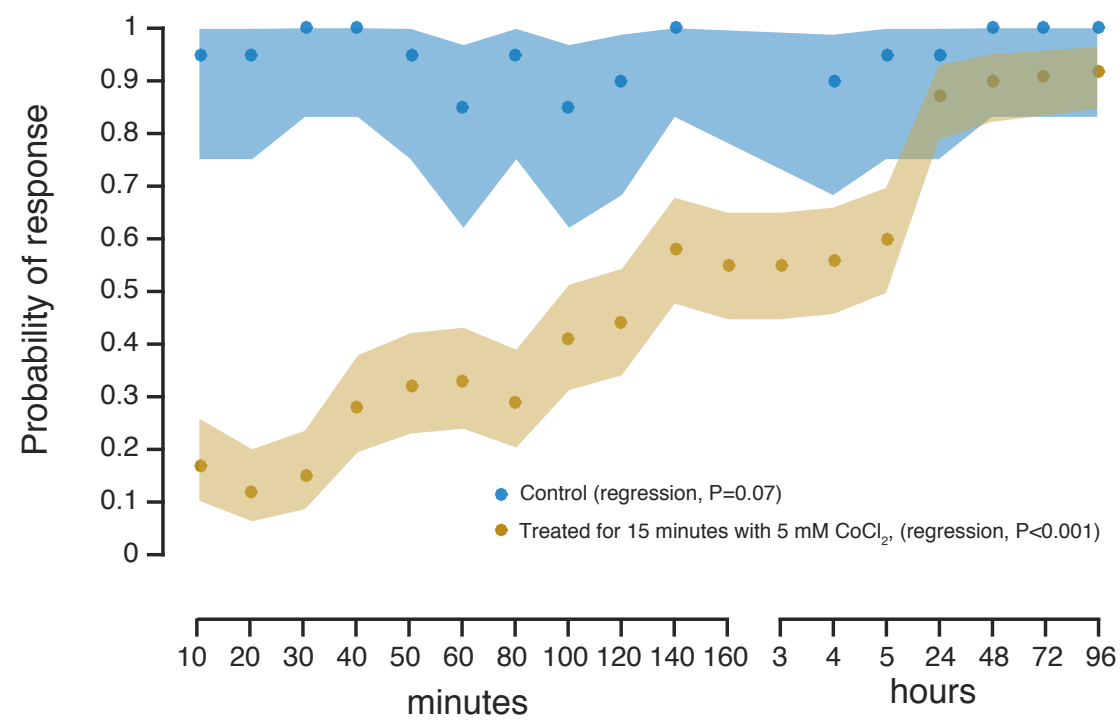

(b) Time after treatment

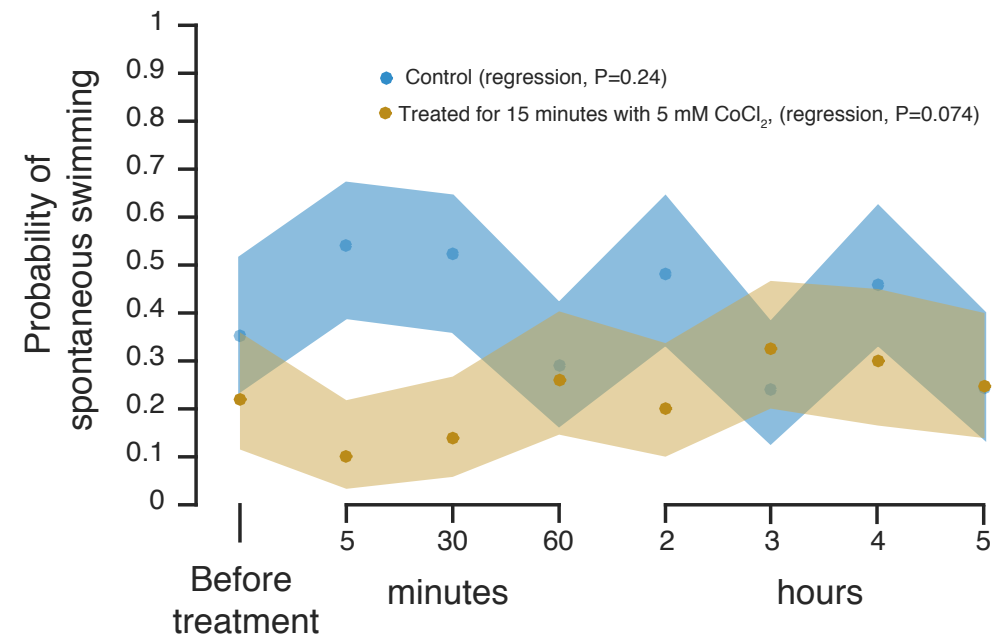

Time after treatment 